\title{
Florística e estrutura do estrato arbóreo de quintais urbanos do município de Belterra, Pará
}

\section{Floristic and structure of the tree layer of urban homegardens of the municipality of Belterra, Pará}

\author{
Breno Pinto Rayol \\ Universidade Federal Rural da Amazônia \\ E-mail: bprayol@yahoo.com.br \\ OrcID: https://orcid.org/0000-0003-2747-2385 \\ Jéssica Conceição Nunes Silva \\ Universidade Federal de Viçosa \\ E-mail: jessicanunes.engflorestal@gmail.com \\ OrclD: https://orcid.org/0000-0002-6436-743X
}

\begin{abstract}
Resumo: Estudos de composição florística e estrutura ampliam o conhecimento de informações ecológicas e botânicas e fornecem subsídios ao manejo e conservação da biodiversidade em agroecossistemas, portanto, o objetivo deste trabalho foi avaliar a florística e a estrutura da vegetação arbórea de quintais urbanos do município de Belterra, Pará. Foram amostrados 19 quintais agroflorestais, nos quais foram inventariados todos os indivíduos arbóreos com diâmetro à altura do peito maior ou igual a cinco centímetros (DAP $\geq 5 \mathrm{~cm}$ ). Foram registradas 503 árvores, distribuídas em 39 espécies e 18 famílias botânicas. As espécies mais importantes na estrutura dos quintais agroflorestais avaliados foram Theobroma grandiflorum (372 indivíduos), Hevea brasiliensis (36) e Persea americana (28). Apesar de se localizarem em áreas urbanas, os quintais avaliados são diversificados e possuem grande importância para o suprimento alimentar de famílias amazônicas.
\end{abstract}

Palavras-chave: Agroecossistemas. Sistemas agroflorestais. Árvores.

Abstract: Floristic composition and structure studies increase the knowledge of ecological and botanical information and provide subsidies for the management and conservation of biodiversity in agroecosystems. Therefore, the objective of this work was to evaluate the floristic and tree vegetation structure of urban homegardens in the municipality of Belterra, Pará. Nineteen homegardens were sampled, in which all tree individuals with a diameter at breast height greater than or equal to five centimeters ( $\mathrm{DBH} \geq 5 \mathrm{~cm}$ ) were inventoried. A total of 503 trees were registered, distributed in 39 species and 18 botanical families. The most important species in the structure of homegardens evaluated were Theobroma grandiflorum (372 individuals), Hevea brasiliensis (36) and Persea americana (28). Despite being located in urban areas, the evaluated homegardens are diversified and have great importance for the food supply of Amazonian families.

Keywords: Agroecosystems. Agroforestry system. Trees.

Data de recebimento: $25 / 05 / 2020$

Data de aprovação: 19/07/2020

DOI: https://doi.org/10.30612/agrarian.v13i50.9369

Introdução

Os quintais agroflorestais são sistemas de uso tradicional dos solos, considerados como uma das formas mais antigas de uso da terra (Florentino et al., 2007). São espaços localizados geralmente em torno das

Agrarian, Dourados, v. 14, n. 51, p. 18-26, 2021. 
residências, onde são cultivadas diversas espécies de múltiplos usos e criados pequenos animais, possuindo como principal função o abastecimento familiar (Kumar \& Nair, 2004). As produções vegetal e animal dos quintais melhoraram o abastecimento de alimentos e colaboram para a nutrição suplementar familiar (Maroyi, 2013; Alemu, 2016), através do aumento da ingestão de alimentos frescos e saudáveis (Aworinde et al., 2013; Pereira \& Figueiredo Neto, 2015), essenciais para a sobrevivência e o bem-estar humano (Mwavu et al., 2016).

Os quintais são caracterizados pela alta diversidade de espécies (Galluzzi et al., 2010) e por abrigarem espécies de diferentes ciclos de vida (Cultrera et al., 2012; Batista \& Barbosa, 2014) que exploram diferentes nichos ecológicos e recursos acima e abaixo do solo (Galluzzi et al., 2010). Esses agroecossistemas domésticos contribuem com a prestação de serviços ecológicos, como serviços de polinização e refúgio para fauna silvestre (Galluzzi et al., 2010; Lope-Alzina \& Howard, 2012), exercendo papéis fundamentais à conservação da diversidade biológica agrícola (Baul et al., 2015).

Apesar dos vários estudos já realizados em quintais agroflorestais na Amazônia (Batista \& Barbosa, 2014; Siviero et al., 2014; Quaresma et al., 2015; Gonçalves \& Lucas, 2017; Lobato et al. 2017), estudos sobre a estrutura desses agroecossistemas são importantes para fornecer subsídios para seu manejo. Portanto, o objetivo deste trabalho foi avaliar a composição florística e a estrutura da vegetação arbórea de quintais urbanos do município de Belterra, ampliando o conhecimento de informações ecológicas, botânicas e de manejo desses agroecossistemas.

\section{Material e Métodos}

\section{Área de estudo}

O estudo foi conduzido no município de Belterra, mesorregião do Baixo Amazonas, situado a cerca de $45 \mathrm{~km}$ da região metropolitana de Santarém, Estado do Pará, Brasil. Encontra-se localizado nas coordenadas $-2^{\circ} 38^{\prime} 11^{\prime \prime} \mathrm{S}$ de latitude e $-54^{\circ} 56^{\prime} 13^{\prime \prime} \mathrm{W}$ de longitude, com altitude é de 176 metros (Figura 1). O clima da região é classificado como Am, segundo a classificação de Koppen, com temperatura e precipitação média anual de $26,2^{\circ} \mathrm{C}$ e $1,743 \mathrm{~mm}$, respectivamente (INMET, 2019). De acordo com Rodrigues et al. (2001), a vegetação predominante é do tipo Ombrófila densa de terra firme e os solos são, em sua maioria, Latossolos ou Argissolos Vermelho e/ou Amarelo de textura argilosa, conforme o sistema brasileiro de classificação de solos (Santos et al., 2018).

\section{Amostragem e coleta de dados}

Foram amostrados 19 quintais agroflorestais localizados na zona urbana do município de Belterra. A seleção dos quintais foi realizada pelo método de amostragem aleatória, com sorteio das ruas amostradas. A área de cada quintal foi mensurada com o auxílio de uma trena de fita flexível. Para avaliação da composição florística e estrutura dos quintais, realizou-se o inventário de todos os indivíduos arbóreos com diâmetro à altura do peito maior ou igual a cinco centímetros (DAP $\geq 5 \mathrm{~cm}$ ). A mensuração do diâmetro foi realizada com foi feita com fita diamétrica e a estimativa da altura com o hipsômetro Forestor Vertex III. 


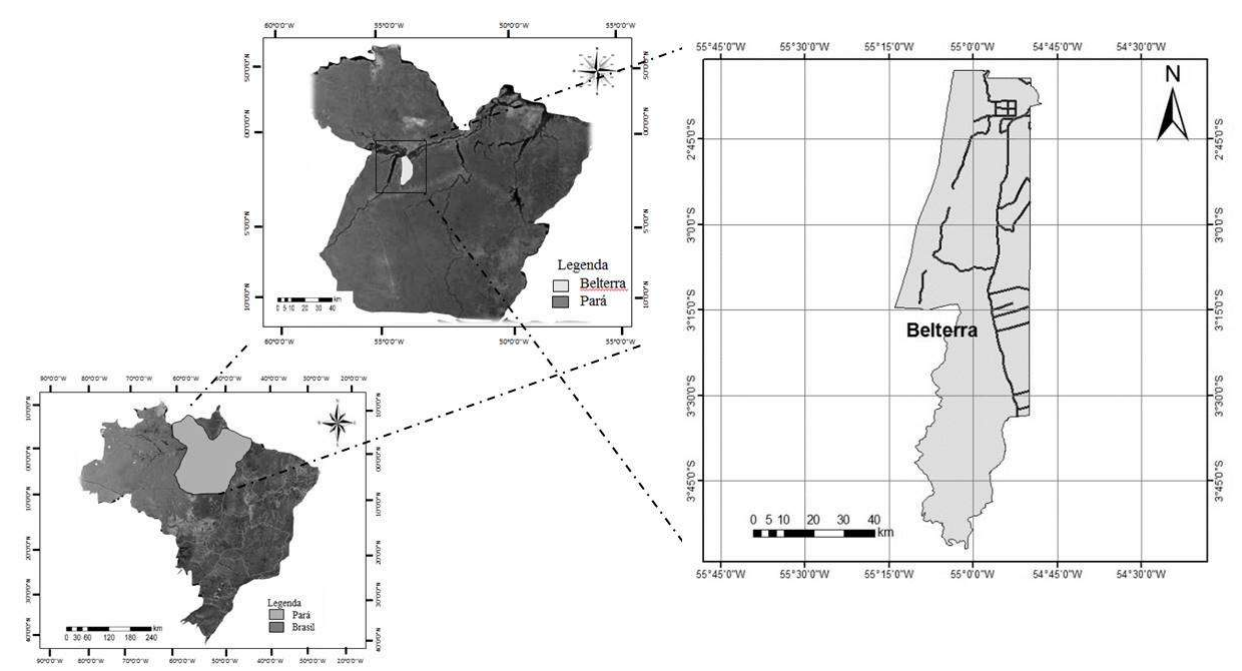

Figura 1. Localização da área de estudo, município de Belterra, estado do Pará, Brasil.

\section{Análise e processamento de dados}

Para a análise de dados dos quintais agroflorestais foi calculada a riqueza de espécies (número de espécies encontradas na área dos quintais agroflorestais). A diversidade foi estimada pelo índice de diversidade de Shannon (H') de acordo com Ludwig e Reynolds (1988). A identificação botânica, quando possível, foi realizada no próprio campo, por meio do reconhecimento das características dendrológicas dos indivíduos. As árvores cujas identificações ficaram duvidosas foram fotografadas e, posteriormente, conferidas pela consulta às chaves taxonômicas e bibliografias especializadas. As espécies foram classificadas de acordo com o uso principal, segundo informações da literatura (Shanley \& Medina, 2005). Para cada espécie foram calculados os parâmetros de densidade (DR), frequência (FR) e dominância (DoR) e valor de importância (IVI), correspondente à soma dos três parâmetros citados anteriormente, conforme Brower et al. (1998).

\section{Resultados e Discussão}

A área média ocupada pelos quintais urbanos avaliados em Belterra foi de $1500 \mathrm{~m}^{2}$. No inventário do estrato arbóreo foram identificados 546 indivíduos, pertencentes a 39 espécies e 18 famílias. As famílias que apresentaram maior riqueza de espécies foram a Rutaceae (5), Anacardiaceae (4) e Arecaceae (4) (Tabela 1). A riqueza média encontrada nos quintais agroflorestais inventariados foi de sete espécies e a abundância média foi de 26 indivíduos por quintal. A diversidade arbórea média, obtida pelo índice de Shannon-Weaver ( $\left.H^{\prime}\right)$, foi de 1,32, valor aproximado de outros quintais da Amazônia (Semedo\& Barbosa, 2007; Batista \& Barbosa, 2014). A diversidade de espécies encontrada nos quintais proporciona variação no provimento da produção durante o ano, contribuindo consequentemente com o sustento de famílias com recursos financeiros limitados (Galluzzi et al., 2010). Famílias com rendas mais baixas são mais propensas a orientar a produção dos seus quintais para o consumo próprio do que famílias com rendas maiores na Amazônia Central (Rayol \& Miranda, 2017), o que confirma a importância desses agroecossistemas como estratégia de segurança e soberania alimentar (Schupp \& Sharp, 2012). 
Tabela 1. Relação de famílias e espécies que ocorrem em quintais agroflorestais do município de Belterra, Oeste do Pará. ( $\mathrm{M}=$ Medicinal; $\mathrm{A}$ = Alimentar; $\mathrm{O}=$ Outros usos).

\begin{tabular}{|c|c|c|c|}
\hline Família & Nome científico & Nome comum & Uso principal \\
\hline \multirow[t]{4}{*}{ Anacardiaceae } & Anacardium occidentale L. & Caju & $\mathrm{A}$ \\
\hline & Caryocar villosum (Albl.) Pers. & Piquiá & $A$ \\
\hline & Mangifera indica L. & Manga & A \\
\hline & Spondias mombim L. & Taperebá & $A$ \\
\hline \multirow[t]{3}{*}{ Annonaceae } & Annona cf montana Macf. & Araticum & $A$ \\
\hline & Annona muricata L. & Graviola & $A$ \\
\hline & Rollinia mucosa (Jacq.) Bail. & Beribá & $A$ \\
\hline Apocynaceae & Himatanthus sucuuba (Spruce ex. Mull. Arg.) & Sucuuba & M \\
\hline \multirow[t]{4}{*}{ Arecaceae } & Bactris gasipaes Kunth & Pupunha & $A$ \\
\hline & Cocos nucifera L. & Coco & A \\
\hline & Euterpe oleraceae Mart. & Açai & $A$ \\
\hline & Oenocarpus bacaba Mart. & Bacaba & $A$ \\
\hline Bignonicaceae & Bixa orellana L. & Urucum & $A$ \\
\hline Caricaceae & Carica papaya L. & Mamão & $A$ \\
\hline Cecropiaceae & Cecropia palmata Willd & Embaúba & $\mathrm{O}$ \\
\hline Chrysobalanaceae & Couepia bracteosa Benth & Pajurá & A \\
\hline \multirow[t]{2}{*}{ Euphorbiaceae } & Croton cajucara Benth. & Sacaca & M \\
\hline & Hevea brasiliensis (HBK) M. Arg. & Seringueira & $A$ \\
\hline \multirow[t]{3}{*}{ Fabaceae } & Bauhinia sp. & Pata de vaca & M \\
\hline & Caesalpinia ferrea Mart. ex Tul. & Jucá & M \\
\hline & Inga edulis Mart. & Ingá & $A$ \\
\hline \multirow[t]{2}{*}{ Lauraceae } & Cinnamomum zeylanicum Blume & Canela & A \\
\hline & Persea americana Mill. & Abacate & A \\
\hline Malpighiaceae & Byrsonima carssifolia H.B.K. & Murici & A \\
\hline \multirow[t]{2}{*}{ Malvaceae } & Theobroma grandiflorum (Willd. Ex Spreng) Schum & Cupuaçu & $A$ \\
\hline & Theobroma cacao L. & Cacau & $A$ \\
\hline \multirow[t]{2}{*}{ Moraceae } & Artocarpus altulis (Sol. ex. Park) Fosb. & Fruta pão & $A$ \\
\hline & Artocarpus heterophyllus Lam. & Jaca & $A$ \\
\hline \multirow[t]{3}{*}{ Myrtaceae } & Eucalyptus globulus Labill & Eucalipto & $A$ \\
\hline & Psidium guajava $\mathrm{L}$. & Goiaba & A \\
\hline & Syzygium malaccence (L.) Merr.\&L.M.Perry & Jambo & $A$ \\
\hline Oxalidaceae & Averrhoa carambola L. & Carambola & $A$ \\
\hline Rosaceae & Rubus urticaefolius Sairet. & Amora & $A$ \\
\hline \multirow[t]{5}{*}{ Rutaceae } & Citrus aurantifolia Swing var.taiti & Limão Taiti & A \\
\hline & Citrus limonia (L.) Osbeck & Limão & $A$ \\
\hline & Citrus medica $\mathrm{L}$. & Cidra & $A$ \\
\hline & Citrus nobilis Lour. var. deliciosa & Tangerina & $A$ \\
\hline & Citrus sinensis (L.) Osbeck & Laranja & A \\
\hline
\end{tabular}

Das espécies amostradas, $86,8 \%$ são para uso alimentar, $10,5 \%$ são usadas como medicinais e $2,6 \%$ categorizadas como outros usos. De acordo com Brienza Júnior et al. (2009) estudos em sistemas agroflorestais sempre tiveram a questão alimentar como um dos principais assuntos abordados. 
As espécies mais importantes nos quintais agroflorestais urbanos avaliados foram Theobroma grandiflorum, Hevea brasiliensis, Persea americana, Mangifera indica e Anacardium occidentale. Essas espécies se destacam tanto em termos de frequência, quanto em densidade e dominância. Juntas correspondem a $75 \%$ da densidade total dos quintais. Essas são espécies muito representativas de quintais agroflorestais amazônicos (Batista \& Barbosa, 2014; Quaresma et al., 2015).

Quanto aos aspectos ecológicos, nos quintais urbanos de Belterra, foram observadas espécies arbóreas ocupando os diferentes estratos, permitindo que a energia solar seja difundida de forma mais eficiente (Saikia et al., 2012). Árvores de grande e médio portes, como seringueira (Hevea brasiliensis), abacateiro (Persea americana), mangueira (Mangifera indica) e cupuaçuzeiro (Theobroma grandiflorum), destacaram-se nos dosséis dos quintais de Belterra como componentes importantes na estrutura vertical desses agroecossistemas.

Apesar da produção dos quintais estar focada principalmente no consumo doméstico, o excedente, em alguns casos, é comercializado, contribuindo para a geração de renda extra (Maroyi, 2013; Aworinde et al., 2013; Alemu, 2016). Nos quintais urbanos de Belterra, também foram observadas espécies de palmeiras, como o açaizeiro e pupunheira, que aliadas à presença do coqueiro, reforçam a grande importância desse grupo na rotina doméstica das populações residentes na Amazônia (Clement et al., 2005).

Tabela 2. Parâmetros fitossociológicos das espécies amostradas no estrato arbóreo dos quintais do município de Belterra, Oeste do Pará. FR= frequência relativa (\%); $D R=$ densidade relativa (\%); DoR= dominância relativa (\%); IVI= índice de valor de importância (\%).

\begin{tabular}{lcccc}
\hline Espécie & FR & DR & DoR & IVI \\
\hline Theobroma grandiflorum (Willd. Ex Spreng) Schum & 14,4 & 50,58 & 27,55 & 92,53 \\
Hevea brasiliensis (HBK) M. Arg. & 4,8 & 6,44 & 20,51 & 31,75 \\
Persea americana Mill. & 8,8 & 6,81 & 11,84 & 27,45 \\
Mangifera indica L. & 5,6 & 5,56 & 10,76 & 21,92 \\
Anacardium occidentale L. & 2,4 & 5,04 & 3,08 & 10,52 \\
Byrsonima carssifolia H.B.K. & 3,2 & 4,33 & 2,29 & 9,82 \\
Citrus sinensis (L.) Osbeck & 6,4 & 1,88 & 1,52 & 9,80 \\
Annona muricata L. & 5,6 & 2,19 & 0,49 & 8,28 \\
Psidium guajava L. & 5,6 & 1,00 & 0,50 & 7,10 \\
Artocarpus heterophyllus Lam. & 1,6 & 2,25 & 2,73 & 6,58 \\
Syzygium malaccence (L.) Merr.\&L.M.Perry & 2,4 & 0,72 & 1,91 & 5,03 \\
Rollinia mucosa (Jacq.) Bail. & 3,2 & 0,64 & 1,19 & 5,03 \\
Citrus nobilis Lour. & 1,6 & 2,7 & 0,68 & 4,98 \\
Carica papaya L. & 3,2 & 0,48 & 0,98 & 4,66 \\
Inga cf. edulis Mart. & 2,4 & 0,91 & 1,33 & 4,64 \\
Eucalyptus globulus Labill & 0,8 & 0,15 & 3,11 & 4,06 \\
Artocarpus altulis (Sol. ex. Park) Fosb. & 2,4 & 0,76 & 0,61 & 3,77 \\
Cocos nucifera L. & 0,8 & 1,05 & 1,63 & 3,48 \\
Spondias mombim L. & 1,6 & 0,3 & 1,53 & 3,43 \\
Bixa orellana L. & 2,4 & 0,48 & 0,27 & 3,15 \\
Couepia bracteosa Benth & 1,6 & 0,36 & 1,15 & 3,11 \\
Cecropia sp. & 1,6 & 0,5 & 0,74 & 2,84 \\
Annona montana Macf. & 1,6 & 0,32 & 0,48 & 2,40 \\
Theobroma cacao L. & 1,6 & 0,37 & 0,3 & 2,27 \\
Averrhoa carambola L. & 1,6 & 0,25 & 0,32 & 2,17 \\
Euterpe oleraceae Mart. & 1,6 & 0,36 & 0,17 & 2,13 \\
& & & &
\end{tabular}


Caesalpinia ferrea Mart.

Bactris gasipaes Kunth

Citrus aurantifolia Swing var. taiti

Citrus limonia (L.) Osbeck

Caryocar villosum (Albl.) Pers.

Rubus urticaefolius Sairet.

Citrus medica L.

Oenocarpus bacaba Mart.

Bauhinia sp.

Himatanthus sucuuba Spruce ex. Mull. Arg.

Croton cajucara Benth.

Cinnamomum zeylanicum Blume

\begin{tabular}{lccc}
1,6 & 0,22 & 0,31 & 2,13 \\
0,8 & 0,7 & 0,48 & 1,98 \\
1,6 & 0,22 & 0,11 & 1,93 \\
0,8 & 0,9 & 0,16 & 1,86 \\
0,8 & 0,23 & 0,43 & 1,46 \\
0,8 & 0,45 & 0,07 & 1,32 \\
0,8 & 0,28 & 0,13 & 1,21 \\
0,8 & 0,14 & 0,21 & 1,15 \\
0,8 & 0,14 & 0,16 & 1,10 \\
0,8 & 0,07 & 0,14 & 1,01 \\
0,8 & 0,07 & 0,11 & 0,98 \\
0,8 & 0,15 & 0,02 & 0,97 \\
\hline $\mathbf{1 0 0}$ & $\mathbf{1 0 0}$ & $\mathbf{1 0 0}$ & $\mathbf{3 0 0}$ \\
\hline
\end{tabular}

\section{Conclusão}

Os quintais urbanos de Belterra são diversificados e caracterizados pela presença de grande quantidade de espécies frutíferas. Isso é um forte indicador da importância desses agroecossistemas para a segurança alimentar e qualidade de vida dos moradores da zona urbana do município.

A variedade de espécies arbóreas reflete na diversificação de produtos oriundos de quintais e consequentemente na contribuição do uso sustentável e manutenção de agrobiodiversidade, portanto, a importância dos quintais na segurança e soberania alimentar de famílias amazônicas, especialmente as com menor renda, indica que políticas públicas nas diferentes esferas devem ser formuladas a fim de estimular sistemas produtivos mais diversificados como estratégia de melhorias da qualidade de vida das populações urbanas.

\section{Agradecimentos}

À Fundação Amazônia Paraense de Amparo à Pesquisa (FAPESPA) pelo apoio financeiro; Às proprietárias e proprietários dos quintais pela cordialidade com que nos receberam em suas residências.

\section{Referências}

Alemu, M.M. (2016). Indigenous agroforestry practices in southern Ethiopia: The case of Lante, Arba Minch. Open Access Library Journal, 3(12), 1-12. http://dx.doi.org/10.4236/oalib.1103278.

Aworinde, D.O., Erinoso, S.M., Ogundairo, B.O., Olanloye, A.O. (2013). Assessment of plants grown and maintained in home gardens in Odeda area Southwestern Nigeria. Journal of Horticulture and Forestry, 5(2), 29-36. http://dx.doi.org/10.5897/JHF2013.0294

Batista, D.L., Barbosa, R.I. (2014). Agrobiodiversidade urbana: composição florística, riqueza e diversidade de plantas nos quintais de Boa Vista, Roraima. Revista Brasileira de Agroecologia, 9(2), 130-150.

Baul, T.K., Rahman, M.M., Moniruzzaman, M., Nandi, R. (2015). Status, utilization, and conservation of agrobiodiversity in farms: a case study in the northwestern region of Bangladesh. International Journal of Biodiversity Science, Ecosystem Services \& Management, 11(4), 318-329. https://doi.org/10.1080/21513732.2015.1050456.

Brienza Júnior, S., Maneschy, R.Q., Mourão Júnior, M., Gazel Filho, A.B., Yared, J.A.G., Gonçalves, D., Gama, M.B.G.B. (2009). Sistemas Agroflorestais na Amazônia Brasileira: Análise de 25 anos de pesquisas. Pesquisa Florestal Brasileira, 60, 67-76. https://doi.org/10.4336/2009.pfb.60.67. 
Brower J.E., Zar, J.H., Van Ende C.N. (1998). Field and laboratory methods for general ecology. WCB/McGraw, New York, 273.

Clement, C.R., Lleras, E., Van Leeuwen, J. (2005). O potencial das palmeiras tropicais no Brasil: acertos e fracassos das últimas décadas. Agrociencia, 9(1-2), 67-71.

Cultrera, M., Amorozo, M.C.M., Ferreira, F.C. (2012). Agricultura urbana e conservação da agrobiodiversidade: um estudo de caso em Mato Grosso, Brasil. Sitientibus. Série Ciências Biológicas, 12(2), 323-332.

Florentino, A.T.N., Araújo, E.L., Albuquerque, U.P. (2007). Contribuição de quintais agroflorestais na conservação de plantas da Caatinga, Município de Caruaru, PE, Brasil. Revista Acta Botanica Brasilica, 21(1), 37-47. https://doi.org/10.1590/S0102-33062007000100005.

Galluzzi, G., Eyzaguirre, P., Negri, V. (2010). Home gardens: neglected hotspots of agro-biodiversity and cultural diversity. Biodiversity and Conservation, 19(13), 3635-3654. https://doi.org/10.1007/s10531-010-9919-5.

Gonçaves, J.P.; Lucas, F.C.A. (2017). Agrobiodiversidade e etnoconhecimento em quintais de Abaetetuba, Pará, Brasil. Revista Brasileira de Biociências, 15(3), 119-134.

Instituto Nacional de Meteorologia (2019). Recuperado em 20 de setembro, 2019, de http://www.inmet.gov.br/portal/index.php?r=bdmep/bdmep.

Kumar, B.M., Nair, P.K.R. (2004). The enigma of tropical homegardens. Agroforestry Systems, 61, 135-152.

Lobato, G.D.J.M., Lucas, F.C.A., Tavares-Martins, A.C.C., Jardim, M.A.G., Martorano, L.G. (2017). Diversidade de uso e aspectos socioambientais de quintais urbanos em Abaetetuba, Pará, Brasil. Revista Brasileira de Agroecologia, 12(2), 95-105.

Lope-Alzina, D.G., Howard, P.L. (2012). The structure, composition, and functions of homegardens: Focus on the Yucatán Peninsula. Etnoecológica, 9(1), 17-41.

Lourenço, J.N.P., Sousa, S.G.A., Wandelli, E.V., Lourenço, F.S., Guimarães, R.R., Campos, L.S.; Silva, R., Martins, V.F.C. (2009). Agrobiodiversidade nos Quintais Agroflorestais em Três Assentamentos na Amazônia Central. Revista Brasileira de Agroecologia, 4(2), 965-969.

Ludwig, J.A., Reynolds, J.F. (1988). Statistical ecology: a primer on methods and computing. New York: John. Wiley \& Sons.

Maroyi, A. (2013). Use and management of homegarden plants in Zvishavane district, Zimbabwe. Tropical Ecology, 54(2), 191-203.

Mwavu, E.N., Ariango, E., Ssegawa, P., Kalema, V.N., Bateganya, F., Waiswa, D., Byakagaba, P. (2016). Agrobiodiversity of homegardens in a commercial sugarcane cultivation land matrix in Uganda. International Journal of Biodiversity Science, Ecosystem Services \& Management, 12(3), 191-201. https://doi.org/10.1080/21513732.2016.1177595.

Quaresma, A.P., Almeida, R.H.C., Oliveira, C.M., Kato, O.R. (2015). Composição florística e faunística de quintais agroflorestais da agricultura familiar no nordeste paraense. Revista Verde de Agroecologia e Desenvo/vimento Sustentável, 10(5), 76-84. https://doi.org/10.18378/rvads.v10i5.3706

Rayol, B.P., Miranda, I.S. (2017). Influência dos fatores socioeconômicos sobre a riqueza de plantas de quintais agroflorestais da Amazônia Central. Revista Espacios, 38(46), 28-40.

Rodrigues, T.E., Santos, P.L., Oliveira Junior, R.C., Valente, M.A., Silva, J.M.L., Cardoso Júnior, E.Q. (2001). Caracterização dos solos da área do planalto de Belterra, município de Santarém, Estado do Pará. [Documentos, № 115]. Belém, PA: Embrapa Amazônia Oriental.

Saikia, P., Choudhury, B.I., Khan, M.L. (2012). Floristic composition and plant utilization pattern in homegardens 
of Upper Assam, India. Tropical Ecology, 53(1), 105-118.

Santos, H.G., Jacomine, P.K.T., Anjos, L.H.C., Oliveira, V.A., Lumbreras, J.F., Coelho, M.R., Almeida, J.A., Araujo Filho, J.C., Oliveira, J.B., Cunha, T.J.F. (2018). Sistema brasileiro de classificação de solos. Brasília: Embrapa.

Schupp, J.L., Sharp, J.S. (2012). Exploring the social bases of home gardening. Agriculture and Human Values, 29(1), 93-105. https://doi.org/10.1007/s10460-011-9321-2.

Semedo, R.J.C.G., Barbosa, R.I. (2007). Árvores frutíferas nos quintais urbanos de Boa Vista, Roraima, Amazônia brasileira. Acta Amazonica, 37(4), 497-504. https://doi.org/10.1590/S0044-59672007000400003. 